\title{
NONNEGATIVE LINEARIZATION OF THE ASSOCIATED $q$-ULTRASPHERICAL POLYNOMIALS
}

\author{
Ryszard Szwarc
}

\begin{abstract}
Nonnegative product linearization of the associated continuous $q$ ultraspherical polynomials is shown for all values $-1<q<1$. This is obtained by applying new criteria for nonnegative product linearization of orthogonal polynomials.
\end{abstract}

\section{Introduction}

Let $\left\{p_{n}\right\}_{n=0}^{\infty}$ be a system of orthogonal polynomials. The product of two of these polynomials can be expressed as a linear combination of these polynomials, i.e., there are coefficients $a(n, m, k)$ such that

$$
p_{n}(x) p_{m}(x)=\sum_{k=|n-m|}^{n+m} a(n, m, k) p_{k}(x) .
$$

If all the coefficients $a(n, m, k)$ are nonnegative, we say that the polynomials $p_{n}$ have nonnegative product linearization.

The problem of which orthogonal polynomials admit nonnegative product linearization has attracted attention for a long time. This is because the property has important consequences, the main one being the convolution structure associated with the polynomials $p_{n}$ determined by the coefficients $a(n, m, k)$ (see $\left.[9,10,22]\right)$. Also, the nonnegativity of the product linearization yields, together with other conditions, certain pointwise estimates of $p_{n}(x)$ (see [22]). In these applications, the knowledge of explicit values of the linearization coefficients is usually not necessary. What counts is their nonnegativity.

The explicit formulas for the linearization coefficients are available only for a small class of orthogonal polynomials. This class includes the ultraspherical polynomials and their $q$-analogues (see $[5,8,12,13,17]$ ). For the Jacobi polynomials $p_{n}^{\alpha, \beta}(x)$, Hylleras [14] set up a recurrence relation involving only three linearization coefficients $a(n, m, k-1), a(n, m, k)$, and $a(n, m, k-1)$, and solved this explicitly for $\alpha=\beta$ (the ultraspherical case) and for $\alpha=\beta+1$. Relying on Hylleras' formula, Gasper [10] has determined the range of values of $\alpha$ and $\beta$ for which nonnegative product linearization holds. Later on, Rahman [17] expressed the linearization coefficients of the Jacobi polynomials as multiples of ${ }_{9} F_{8}$ hypergeometric series from which their nonnegativity was obvious for $\alpha \geq \beta>-1$ and $\alpha+\beta+1 \geq 0$, a substantial part of the set which Gasper $[9,10]$ found in 1970 . He managed to extend this to $q$-analogues of the Jacobi polynomials and proved nonnegativity of the product linearization for $\alpha \geq \beta$, $\alpha+\beta+1 \geq 0$, and $0 \leq q<1$. Gasper [11] used Rahman's ${ }_{10} \varphi_{9}$-series representation [17] of the linearization coefficients of $q$-Jacobi polynomials and determined the range of $\alpha$ and $\beta$ for which the linearization coefficients are nonnegative.

Received December 5, 1994, revised April 19, 1995.

Supported by Committee for Scientific Research (Poland) under grant KBN 2 P03A 03009. 
Another approach is to study general orthogonal polynomials in order to find conditions for nonnegative product linearization and then test them on specific orthogonal polynomials. The first result of this kind belongs to Askey [1], and it can be applied to a wide class of Jacobi polynomials and their $q$-analogues. However, it does not cover the whole range of parameters $\alpha$ and $\beta$, and some important special cases like the Legendre polynomials are left open. In 1992, in two papers [20, 21], new conditions were found which when applied to the Jacobi polynomials come close to Gasper's result since they give nonnegative product linearization for $\alpha \geq \beta>-1, \alpha+\beta+1 \geq 0$. These conditions are given in terms of the coefficients in the three-term recurrence relation that the orthogonal polynomials satisfy. Roughly, some monotonicity properties of these coefficients are required. Unfortunately, these conditions do not admit coefficients oscillating about certain values. However, there is one important instance where such oscillating behavior occurs: the $q$-ultraspherical polynomials with $q$ negative.

The aim of this paper is to give new criteria for the nonnegative product linearization of orthogonal polynomials in order to handle oscillating coefficients in the three-term recurrence relation. These results are collected in Section 3. The criteria then are applied to the associated $q$-ultraspherical polynomials to show that they have nonnegative product linearization for all values of $q$, including $q$ negative (the case of positive $q$ has been partially solved by Bustoz and Ismail [6, Thm. 2.3] by applying the aforementioned criterion of Askey). This is done in Section 2.

\section{Associated $q$-ultraspherical polynomials}

Let $\mu$ be a probability measure $\mu$ with infinite support on the real line and symmetric about the origin, i.e., $\mu(A)=\mu(-A)$ for any Borel subset of $A$ reals. Let $\left\{p_{n}(x)\right\}_{n=0}^{\infty}$ be a system of polynomials, orthonormal with respect to $\mu$. We assume that the polynomials $p_{n}(x)$ have positive leading coefficients. Then they satisfy the recurrence relation

$$
x p_{n}(x)=\lambda_{n} p_{n+1}(x)+\lambda_{n-1} p_{n-1}(x), \quad n \geq 0
$$

with the convention $p_{-1}=\lambda_{-1}=0$. The coefficients $\lambda_{n}$ are strictly positive as the ratios of the leading coefficients of $p_{n}(x)$ and $p_{n+1}(x)$.

The associated polynomials $p_{n}(x ; k)$ of order $k$ are defined for any nonnegative integer $k$ by the recurrence formula

$$
x p_{n}(x ; k)=\lambda_{n+k} p_{n+1}(x ; k)+\lambda_{n+k-1} p_{n-1}(x ; k), \quad n \geq 0,
$$

with $p_{-1}(x ; k)=0$. If $\lambda_{n}$ is a rational function of $n$ or of $q^{n}$, then we can admit $k$ real, as long as $n+k$ is not a singular point of the function $z \mapsto \lambda_{z}$. The associated polynomials $p_{n}(x ; 1)$ of order 1 also are called the numerator polynomials, and they turn up naturally when considering a continued fraction approximation of the Stieltjes transform of the measure $\mu$ (see [7, Chapter III.4] ).

The associated polynomials again are orthonormal with respect to a probability measure on $\mathbb{R}$. Although this new measure is determined by the original measure $\mu$, the relation is rather intricate, and one cannot expect that the new measure can be found explicitly in terms of $\mu$.

For a sequence of positive numbers $\left\{\sigma_{n}\right\}_{n=0}^{\infty}$, let

$$
q_{n}(x)=\sigma_{n}^{-1} p_{n}(x)
$$


We call $q_{n}(x)$ the renormalized polynomials. The renormalized polynomials $q_{n}(x)$ are no longer orthonormal, and they satisfy a new recurrence relation

$$
\begin{aligned}
& x q_{n}(x)=\gamma_{n} q_{n+1}(x)+\alpha_{n} q_{n-1}(x) \\
& \text { where } \quad \gamma_{n}=\frac{\sigma_{n+1}}{\sigma_{n}} \lambda_{n}, \quad \alpha_{n}=\frac{\sigma_{n-1}}{\sigma_{n}} \lambda_{n-1}, \quad n \geq 0,
\end{aligned}
$$

with the condition $\sigma_{-1}=0$. Thus, the coefficients $\alpha_{n}, \gamma_{n}$ are positive (except for $\left.\alpha_{0}=0\right)$, and they are related to $\lambda_{n}$ by

$$
\lambda_{n}^{2}=\alpha_{n+1} \gamma_{n}
$$

Conversely, if two sequences of positive numbers $\left\{\alpha_{n}\right\}_{n=1}^{\infty}$ and $\left\{\gamma_{n}\right\}_{n=0}^{\infty}$ satisfy (5), then the polynomials $q_{n}$, defined recursively by (4), are the renormalized polynomials. Indeed, the numbers $\sigma_{n}$ can be given as

$$
\sigma_{n}^{2}=\frac{\gamma_{0} \gamma_{1} \cdots \gamma_{n-1}}{\alpha_{1} \alpha_{2} \cdots \alpha_{n}}
$$

In this way, Theorem 1 of [21] can be restated as

Theorem 1. Let the polynomials $p_{n}(x)$ satisfy (2). Assume there are sequences of numbers $\alpha_{n}$ and $\gamma_{n}$, positive except for $\alpha_{0}=0$, such that for $n \geq 0$

(i) $\lambda_{n}^{2}=\alpha_{n+1} \gamma_{n}$,

(ii) $\alpha_{n} \leq \alpha_{n+2}$

(iii) $\alpha_{n}+\gamma_{n} \leq \alpha_{n+2}+\gamma_{n+2}$,

(iv) $\alpha_{n} \leq \gamma_{n}$.

Then the polynomials $p_{n}(x)$ have nonnegative product linearization.

If it happens that $\lambda_{n}$ itself is increasing, then we can apply Askey's theorem [1] in order to get nonnegative product linearization. In that case, Theorem 1 also can be applied with $\alpha_{n+1}=\gamma_{n}=\lambda_{n}$.

We now turn to considering the $q$-ultraspherical polynomials. Part of the result will be obtained by using Askey's criterion, as it was done in [6]. However, this criterion does not work when $q$ is negative. Moreover, it does not apply to all cases when $q$ is positive. In these cases, we will have to use other methods based on Theorem 1 .

The $q$-ultraspherical polynomials were introduced and studied by Rogers [18]. They have two parameters $q$ and $\beta$. We will assume that both parameters are real and of absolute value less than 1 . In the standard normalization, the $q$-ultraspherical polynomials are denoted by $C_{n}(x ; \beta \mid q)$, and they satisfy the recurrence relation,

$$
2 x C_{n}(x ; \beta \mid q)=\frac{1-q^{n+1}}{1-\beta q^{n}} C_{n+1}(x ; \beta \mid q)+\frac{1-\beta^{2} q^{n-1}}{1-\beta q^{n}} C_{n-1}(x ; \beta \mid q)
$$

for $n \geq 0$, with $C_{-1}(x ; \beta \mid q)=0$. The orthogonality measure is known explicitly (see [4, Thm. 2.2 and Sect. 4] or [13, Sect. 7.4]). When $|\beta|,|q|<1$, it is absolutely continuous with respect to Lebesgue measure on the interval $[-1,1]$. Later, we will make use of the fact that the support of this measure is contained in $[-1,1]$ in one of the cases we will be considering.

The linearization coefficients of the $q$-ultraspherical polynomials were already computed explicitly by Rogers [18] (see also [5, 12, 17]). They are given as products of factors which can be readily checked to be positive when $|\beta|,|q|<1$. However, no explicit formula is available for the linearization coefficients of the polynomials associated with $q$-ultraspherical polynomials. 
In order to handle the associated $q$-ultraspherical polynomials following [6], we add one more real parameter $\alpha$ from the interval $[-1,1]$ and define the polynomials $C_{n}^{\alpha}(x ; \beta \mid q)$ by the recurrence relation

$$
\begin{aligned}
2 x C_{n}^{\alpha}(x ; \beta \mid q)= & \frac{1-\alpha q^{n+1}}{1-\beta \alpha q^{n}} C_{n+1}^{\alpha}(x ; \beta \mid q) \\
& \quad+\frac{1-\beta^{2} \alpha q^{n-1}}{1-\beta \alpha q^{n}} C_{n-1}^{\alpha}(x ; \beta \mid q) .
\end{aligned}
$$

Observe that $C_{n}^{q^{k}}(x ; \beta \mid q)$, where $k \geq 0$, are just the associated $q$-ultraspherical polynomials. These polynomials were first introduced in [6], where the orthogonality relation is given explicitly.

The polynomials $C_{n}^{\alpha}(x ; \beta \mid q)$ are not orthonormal. By (4) and (5), the orthonormal q-ultraspherical polynomials $\widehat{C}_{n}^{\alpha}(x ; \beta \mid q)$ satisfy (2) with

$$
\lambda_{n}^{2}=\lambda_{n}^{2}(\alpha)=\frac{\left(1-\alpha q^{n+1}\right)\left(1-\beta^{2} \alpha q^{n}\right)}{4\left(1-\beta \alpha q^{n}\right)\left(1-\beta \alpha q^{n+1}\right)} .
$$

Then one can compute that

$$
\lambda_{n+1}^{2}(\alpha)-\lambda_{n}^{2}(\alpha)=\frac{\alpha(q-\beta) q^{n}(1-q)(1-\beta)\left(1+\alpha \beta q^{n+1}\right)}{4\left(1-\beta \alpha q^{n}\right)\left(1-\beta \alpha q^{n+1}\right)\left(1-\beta \alpha q^{n+2}\right)} .
$$

The next theorem is known for $0<q<1$ and $\alpha(q-\beta) \geq 0$ [6, Thm. 2.3]. The main novelty here is contained in part (i) when the coefficients in the recurrence formula are oscillating. Also, we show that for $\alpha$ nonnegative, no restrictions on the other two parameters are required.

Theorem 2. Let $|\beta|,|q|<1$, and $|\alpha| \leq 1$. Assume one of the following conditions is satisfied

(i) $q \leq 0$,

(ii) $\alpha \geq 0$,

(iii) $\alpha<0, q>0$, and $\beta>q^{2}$.

Then the polynomials $C_{n}^{\alpha}(x ; \beta \mid q)$ have nonnegative product linearization.

Proof. We will break the proof into two cases.

(a) $-1<q<0$. This is the most interesting case, since the coefficients $\lambda_{n}(\alpha)$ are oscillating about $\frac{1}{2}$. Indeed, it can be shown that

$$
\lambda_{n}^{2}(\alpha)=\frac{1}{4}+\frac{1}{4} \frac{q^{n} \alpha(\beta-q)(1-\beta)}{\left(1-\alpha \beta q^{n}\right)\left(1-\alpha \beta q^{n+1}\right)} .
$$

Such behavior has never been studied before for general orthogonal polynomials in connection with nonnegative product linearization.

We will apply Theorem 3. For this purpose, we will need the following formulas that can be readily checked,

$$
\begin{gathered}
\lambda_{m+2}^{2}(\alpha)-\lambda_{m}^{2}(\alpha)=\frac{\alpha(q-\beta) q^{m}\left(1-q^{2}\right)(1-\beta)\left(1-\alpha^{2} \beta^{2} q^{2 m+3}\right)}{4\left(1-\alpha \beta q^{m}\right)\left(1-\alpha \beta q^{m+1}\right)\left(1-\alpha \beta q^{m+2}\right)\left(1-\alpha \beta q^{m+3}\right)}, \\
\left\{\lambda_{m+3}^{2}(\alpha)+\lambda_{m+2}^{2}(\alpha)\right\}-\left\{\lambda_{m+1}^{2}(\alpha)+\lambda_{m}^{2}(\alpha)\right\} \\
=\frac{\alpha(q-\beta) q^{m}\left(1-q^{2}\right)(1+q)(1-\beta)\left(1+\alpha \beta q^{m+2}\right)}{4\left(1-\alpha \beta q^{m}\right)\left(1-\alpha \beta q^{m+2}\right)\left(1-\alpha \beta q^{m+4}\right)}
\end{gathered}
$$

Now, if $\alpha(q-\beta) \leq 0$, set $m=2 n-1$ and apply Theorem 3(i). Otherwise, set $m=2 n$ and apply Theorem 3(ii). 
(b) $0<q<1$. Assume $\alpha(q-\beta) \geq 0$. By (9), the sequence $\lambda_{n}(\alpha)$ is nondecreasing. In this case, by Askey's theorem [1] or by Theorem 1 with $\alpha_{n+1}=\gamma_{n}=\lambda_{n}(\alpha)$, we get the conclusion.

Now let $\alpha(q-\beta)<0$. First consider the case $\alpha>0$ and $q<\beta$. Then by (9), the sequence $\lambda_{n}(\alpha)$ is decreasing and it tends to $\frac{1}{2}$. In view of Proposition 1 , it suffices to show that the polynomials $C_{n}^{\alpha}(x ; \beta \mid q)$ take positive values at $x=1$. It can be computed that

$$
\lambda_{n}^{2}(1)-\lambda_{n}^{2}(\alpha)=\frac{q^{n}(\beta-q)(1-\beta)(1-\alpha)\left(1-\alpha \beta^{2} q^{2 n+1}\right)}{4\left(1-\alpha \beta q^{n}\right)\left(1-\alpha \beta q^{n+1}\right)\left(1-\beta q^{n}\right)\left(1-\beta q^{n+1}\right)} \geq 0 .
$$

Thus by Lemma 1 and Proposition 1, we are reduced to showing that the polynomials $C_{n}(x ; \beta \mid q)$, i.e., the $q$-ultraspherical polynomials, take positive values at $x=1$. The latter follows from the fact that the orthogonality measure for these polynomials is supported in the interval $[-1,1]$ (see [4, Theorem 2.2]).

In order to complete the proof, assume $\alpha<0$ and $q^{2} \leq \beta<q$. By (9), the sequence $\lambda_{n}(\alpha)$ is decreasing and it tends to $\frac{1}{2}$. By [6, Thm. 2.2] the orthogonality measure is supported in the interval $[-1,1]$. This implies the corresponding polynomials take positive values at $x=1$. Now we can apply Proposition 1 .

Remark 1. The $q^{2}$ in (iii) cannot be replaced by any value $\beta_{0}<q^{3}$. This is because, by $[6, \mathrm{Thm} .2 .1]$, the orthogonality measure has a mass at the right end of its support. This contradicts nonnegative linearization (see [19, Thm. 6 (iii)]).

Remark 2. Part (ii) of Theorem 2 gives an affirmative answer to a conjecture of Askey (see [6, p. 728]), who suspected that nonnegative linearization holds for $0<$ $q<1,0<\alpha<1$, and $0<\beta<1$.

Since, by (8), we have

$$
\lambda_{n}(\alpha) \rightarrow \frac{1}{2}
$$

the support of the orthogonality measure consists of the interval $[-1,1]$ and at most countably many mass points off this interval. Assume this countable set is nonempty. Since the support is symmetric about the origin, the right end of it is a mass point. By virtue of the aforementioned [19, Thm. 6 (iii)], the corresponding polynomials cannot have nonnegative linearization. Hence, the proof of Theorem 2 gives the following.

Corollary 1. The polynomials $C_{n}^{\alpha}(x ; \beta \mid q)$ have nonnegative product linearization if and only if the orthogonality measure is supported in the interval $[-1,1]$.

By (9), the coefficients $\lambda_{n}(\alpha)$ tend to $\frac{1}{2}$ fast enough to satisfy Nevai's theorem [16, Thm. 40, p.143]. This combined with Theorem 2 and Corollary 1 implies

Corollary 2 ([6] Thm. 2.2). Let $q, \alpha$, and $\beta$ satisfy the assumptions of Theorem 2. Then the orthogonality measure of the polynomials $C_{n}^{\alpha}(x ; \beta \mid q)$ has no mass points and its support coincides with the interval $[-1,1]$.

For nonnegative integer $k$, set $\alpha=q^{k}$. Then the polynomials $C_{n}^{\alpha}(x ; \beta \mid q)$ are the associated $q$-ultraspherical polynomials of order $k$. Thus we obtain

Corollary 3. The associated q-ultraspherical polynomials of any order have nonnegative product linearization for $|q|,|\beta|<1$. 


\section{Auxiliary general results.}

In this section, we collect results of a general nature that have been used in Section 2 for the associated $q$-ultraspherical polynomials.

Lemma 1. Let $p_{n}(x)$ and $q_{n}(x)$ satisfy

$$
\begin{aligned}
& x p_{n}(x)=\lambda_{n} p_{n+1}(x)+\lambda_{n-1} p_{n-1}(x), \\
& x q_{n}(x)=\lambda_{n}^{\prime} q_{n+1}(x)+\lambda_{n-1}^{\prime} q_{n-1}(x),
\end{aligned}
$$

where $\lambda_{n}, \lambda_{n}^{\prime}$ are sequences of positive numbers such that $\lambda_{n}^{\prime} \leq \lambda_{n}$. Assume $p_{n}(1)>0$ for $n \geq 0$. Then $q_{n}(1)>0$, for $n \geq 0$.

Proof. Let $\mu$ and $\mu^{\prime}$ denote the orthogonality measures associated with $p_{n}(x)$ and $q_{n}(x)$, respectively. These measures are symmetric about the origin. Since $p_{n}(1)>0$, the support of $\mu$ is located to the left of 1 . By symmetry, it is contained in the interval $[-1,1]$. We will show that the support of $\mu^{\prime}$ also is contained in $[-1,1]$.

Observe that by (10), the $n$th moment of $\mu$, i.e., the integral $\int x^{n} d \mu(x)$ is a polynomial of $\lambda_{0}, \lambda_{1}, \ldots$ with nonnegative coefficients. Thus,

$$
\int_{-\infty}^{\infty} x^{n} d \mu^{\prime}(x) \leq \int_{-\infty}^{\infty} x^{n} d \mu(x),
$$

but

Therefore,

$$
\int_{-\infty}^{\infty} x^{n} d \mu(x)=\int_{-1}^{1} x^{n} d \mu(x) \leq 1 .
$$

$$
\int_{-\infty}^{\infty} x^{n} d \mu^{\prime}(x) \leq 1
$$

This implies $\operatorname{supp} \mu \subset[-1,1]$. As a consequence, we obtain $q_{n}(1)>0$.

Proposition 1. Assume the $p_{n}$ satisfy (2) with $\lambda_{n} \geq \frac{1}{2}$ for $n \geq 0$. If $p_{n}(1)>0$ for $n \geq 0$, the polynomials $p_{n}$ have nonnegative product linearization.

Proof. On substituting $x=1$ into (2) and dividing by $p_{n}(1)$, we get

$$
\lambda_{n} \frac{p_{n+1}(1)}{p_{n}(1)}+\lambda_{n-1} \frac{p_{n-1}(1)}{p_{n}(1)}=1 \text {. }
$$

Hence,

$$
\lambda_{n}^{2}=\alpha_{n+1}\left(1-\alpha_{n}\right)
$$

where

$$
\alpha_{n}=\lambda_{n-1} \frac{p_{n-1}(1)}{p_{n}(1)} .
$$

By assumption, $\alpha_{n}>0$. Thus (12) implies $\alpha_{n}<1$, and we get

$$
\alpha_{n+1}\left(1-\alpha_{n}\right)>\frac{1}{4} \geq \alpha_{n}\left(1-\alpha_{n}\right)
$$

so that $\alpha_{n+1} \geq \alpha_{n}$. Therefore, $\alpha_{n}$ has a limit, say $\alpha$. By (12), the sequence $\lambda_{n}$ also is convergent, say to $\lambda \geq \frac{1}{2}$, and

$$
\alpha(1-\alpha)=\lambda^{2} \geq \frac{1}{4}
$$

This yields $\alpha=\frac{1}{2}$.

Put $\gamma_{n}=1-\alpha_{n}$, then, by (12),

$$
\lambda_{n}^{2}=\alpha_{n+1} \gamma_{n}
$$


We claim that $\alpha_{n}$ and $\gamma_{n}$ satisfy the assumptions of Theorem 1 . Indeed, the sequences $\alpha_{n}$ and $\alpha_{n}+\gamma_{n}=1$ are nondecreasing. Also, since $\alpha_{n} \nearrow \frac{1}{2}$, we obtain

$$
\gamma_{n}=1-\alpha_{n} \geq \frac{1}{2} \geq \alpha_{n}
$$

This completes the proof of the proposition.

Theorem 3. Assume the $p_{n}(x)$ satisfy (2) and either (i) or (ii) is satisfied, where

(i) the sequences $\lambda_{2 n-1}^{2}, \lambda_{2 n-1}^{2}+\lambda_{2 n}^{2}$ are nondecreasing and $\lambda_{2 n-1}^{2} \leq \lambda_{2 n}^{2}$,

(ii) the sequences $\lambda_{2 n}^{2}, \lambda_{2 n}^{2}+\lambda_{2 n+1}^{2}$ are nondecreasing and $\lambda_{2 n}^{2} \leq \lambda_{2 n+1}^{2}$.

Then the polynomials $p_{n}(x)$ admit nonnegative product linearization.

Proof. In the case (i), set

$$
\begin{gathered}
\alpha_{2 n+1}=\gamma_{2 n+1}=1, \\
\alpha_{2 n}=\lambda_{2 n-1}^{2}, \quad \gamma_{2 n}=\lambda_{2 n}^{2},
\end{gathered}
$$

for $n \geq 0$. We assume that $\alpha_{0}=\lambda_{-1}=0$. Then

$$
\begin{gathered}
\alpha_{2 n+1}+\gamma_{2 n+1}=2, \\
\alpha_{2 n}+\gamma_{2 n}=\lambda_{2 n-1}^{2}+\lambda_{2 n}^{2},
\end{gathered}
$$

for $n \geq 0$. Thus, the assumptions of Theorem 1 are satisfied.

In the case (ii), set

$$
\begin{gathered}
\alpha_{0}=0, \quad \gamma_{1}=1, \\
\alpha_{2 n}=\gamma_{2 n}=1, \\
\alpha_{2 n+1}=\lambda_{2 n}^{2}, \quad \gamma_{2 n+1}=\lambda_{2 n+1}^{2}, \quad n \geq 0 .
\end{gathered}
$$

Then

$$
\begin{gathered}
\alpha_{0}+\gamma_{0}=1, \\
\alpha_{2 n}+\gamma_{2 n}=2, \\
\alpha_{2 n+1}+\gamma_{2 n+1}=\lambda_{2 n}^{2}+\lambda_{2 n+1}^{2}, \quad n \geq 0 .
\end{gathered}
$$

Again, the assumptions of Theorem 1 are satisfied.

Remark 3. It is worth observing that in case (ii) we can slightly weaken the assumption due to the fact that $\alpha_{0}+\gamma_{0}=1<2$. Namely, we can change the values of $\gamma_{0}$ and $\alpha_{1}$ by setting

$$
\gamma_{0}=2, \quad \alpha_{1}=\frac{1}{2} \lambda_{0}^{2}
$$

The assumptions of Theorem 1 remain valid. Therefore, it suffices to assume that the new sequence $\tilde{\lambda}_{n}$ satisfies (ii), where

$$
\tilde{\lambda}_{0}^{2}=\frac{1}{2} \lambda_{0}^{2}, \quad \tilde{\lambda}_{n}^{2}=\lambda_{n}^{2}, \quad n \geq 1 .
$$

Corollary 4. Let $p_{n}(x)$ satisfy (2) with

$$
\lambda_{n}^{2}=\frac{1}{4}+(-1)^{n} \varepsilon \theta_{n}
$$

where $\varepsilon= \pm 1$ and $\theta_{n}$ is a convex nonincreasing sequence of positive numbers. Then the polynomials $p_{n}(x)$ have nonnegative product linearization. 
Proof. Let $\varepsilon=1$. Then the assumptions of Theorem 3(i) are satisfied. Indeed, the sequence $\lambda_{2 n-1}^{2}=\frac{1}{4}-\theta_{2 n-1}$ is nondecreasing. Moreover,

$$
\lambda_{2 n}^{2}=\frac{1}{4}+\theta_{2 n} \geq \frac{1}{4}-\theta_{2 n-1}=\lambda_{2 n-1}^{2} .
$$

Finally, the sequence

$$
\lambda_{2 n-1}^{2}+\lambda_{2 n}^{2}=\frac{1}{2}-\theta_{2 n-1}+\theta_{2 n}
$$

is nondecreasing, as $\theta_{n}$ is a convex sequence.

Similarly, we apply Theorem 3(ii) for $\varepsilon=-1$.

Acknowledgment. I would like to thank Mourad Ismail for suggesting the use of the independent parameter in order to handle the associated polynomials and for pointing out the references $[3,6,15]$ to me.

\section{References}

1. R. Askey, Linearization of the product of orthogonal polynomials, Problems in Analysis, (R. Gunning, ed.), Princeton University Press, Princeton, New Jersey, 1970, 223-228.

2. Lrthogonal Polynomials and Special Functions, Regional Conference Series in Applied Mathematics 21, Society for Industrial and Applied Mathematics, Philadelphia, Pennsylvania, 1975.

3. R. Askey and M. E. H. Ismail, A generalization of ultraspherical polynomials, In: Studies in Pure Mathematics, P. Erdös, ed., Birkhäuser, Basel, 1983, pp.55-78.

4. R. Askey and J. A. Wilson, Some basic hypergeometric orthogonal polynomials that generalize Jacobi polynomials, Mem. Amer. Math. Soc. 54 (1985).

5. D. M. Bressoud, Linearization and related formulas for q-ultraspherical polynomials, SIAM J. Math. Anal. 12 (1981), 161-168.

6. J. Bustoz and M. E. H. Ismail, The associated ultraspherical polynomials and their q-analogues, Canad. J. Math. 34 (1982), 718-736.

7. T. Chihara, An Introduction to Orthogonal Polynomials, Vol. 13, Mathematics and Its Applications, Gordon and Breach, New York, London, Paris, 1978.

8. J. Dougall, A theorem Sonine in Bessel functions, with two extensions to spherical harmonics, Proc. Edinburgh Math. Soc. 37 (1919), 33-47.

9. G. Gasper, Linearization of the product of Jacobi polynomials I, Canad. J. Math., 22 (1970), 171-175.

10. Linearization of the product of Jacobi polynomials II, Canad. J. Math., 22 (1970), 582593.

11. tinuous q-Jacobi polynomials, In: Conference on Harmonic Analysis in Honor of Antoni Zygmund (1983), Wadsworth International Group, Belmont, Calif., 44-59.

12. , Rogers' linearization formula for the continuous q-ultraspherical polynomials and quadratic transformation formulas, SIAM J. Math. Anal. 16 (1985), 1061-1071.

13. G. Gasper and M. Rahman, Basic Hypergeometric Series, Vol. 35, Encyclopedia of Mathematics and Its Applications, Cambridge University Press, Cambridge, 1990.

14. E. Hylleras, Linearization of products of Jacobi polynomials, Math. Scand. 10 (1962), 189-200.

15. C. Markett, Linearization of the product of symmetric orthogonal polynomials, Constr. Approx. 10 (1994), 317-338.

16. P. Nevai, Orthogonal Polynomials, Mem. Amer. Math. Soc. 213 (1979).

17. M. Rahman, The linearization of the product of continuous q-Jacobi polynomials, Can. J. Math. 33 (1981), 961-987.

18. L. J. Rogers, Second memoir on the expansion of certain infinite products, Proc. London Math. Soc. 25 (1894), 318-343.

19. A. L. Schwartz, $\ell^{1}$-convolution algebras: representation and factorization, $\mathrm{Z}$. Wahrsch. Verw. Gebiete 41 (1977), 161-176. 
20. R. Szwarc, Orthogonal polynomials and a discrete boundary value problem, I, SIAM J. Math. Anal. 23 (1992), 959-964.

21. __ Orthogonal polynomials and a discrete boundary value problem, II Ibid 23 (1992), 965969.

22. _ Convolution structures associated with orthogonal polynomials, J. Math. Anal. Appl. 170 (1992), 158-170.

Institute of Mathematics, Wroclaw University, Pl. Grunwaldzki 2/4, 50-384 Wroclaw, POLAND

E-mail: szwarc@math.uni.wroc.pl 